\title{
Endobronchial Malposition of Matthys Pleural Catheter: A Case Report
}

\author{
Andreas Kirschbaum ${ }^{1} \quad$ Alexander Damanakis ${ }^{1}$ Caroline Rolfes ${ }^{2}$ Detlef Bartsch ${ }^{1}$ \\ 1 Department of Surgery, University Hospital, Marburg, Germany \\ 2 Department of Anaesthesiology, University Hospital, Marburg, \\ Germany \\ Address for correspondence Andreas Kirschbaum, MD, Department \\ of Surgery, University Hospital, Baldingerstrasse, Marburg 35033 , \\ Germany (e-mail: Kirschbaum001@gmx.de).
}

Thorac Cardiovasc Surg Rep 2015;4:14-17.
Abstract
Keywords
- pleural catheter
- pleural effusion
- Matthys catheter
- malposition
- complication

Background Matthys catheters (Matthys drainage A Set, Bösch Feinmechanik und Medizintechnik $\mathrm{GmbH}$, Gottenheim, Germany) are thin catheters with an external diameter of $2.7 \mathrm{~mm}$, which are often used in internal medicine to drain pleural effusions. After puncturing the pleural cavity with a hollow needle the Matthys catheter is advanced through it without resistance. Once the three-way tap on the catheter has been opened, the pleural effusion should flow out.

Case Description In our case, the positioning of the Matthys catheter was thought to be completely straightforward but an X-ray check nonetheless revealed malposition. The catheter had been advanced surprisingly over the left upper lobe bronchus across the carina into the right upper lobe bronchus. No thoracic bleeding occurred.

Conclusion Although the Matthys catheter was placed endobronchially, we removed it without air leakage. Fortunately, no further problems occurred.

\section{Introduction}

Pleural effusions are very common. They can have very varied causes ranging from cardiac insufficiency to inflammatory changes of the pleura. ${ }^{1}$ Above a certain amount, the lung becomes compromised. Affected individuals experience breathlessness and a reduction in physical performance depending on their reserves of cardiopulmonary function. In this situation, relief of the pressure is indicated. ${ }^{2}$ This can be achieved by means of one or several pleural punctures or by inserting a drain which is then left in place. If the pleural effusion is persistent and rapid, drain insertion is recommended.

In internal medicine, a so-called Matthys catheter (Matthys Drainage A Set, Bösch Feinmechanik und Medizintechnik $\mathrm{GmbH}$, Gottenheim, Germany)-a silicone catheter with an external diameter of $2.7 \mathrm{~mm}$-is often used for this purpose. ${ }^{3}$ The pleural cavity is punctured with a hollow needle under ultrasound control. Over this hollow needle, the catheter will be pushed into the pleural cavity without resistance. With the appropriate technique, the risk of injury to the intercostal artery is very low. If the pleural effusion is not serous but thicker or loculated, a thoracic drain should be inserted. However, pleural effusions that are purely serous can be dealt with very well and successfully with a Matthys catheter.

In spite of the simple insertion technique, grotesque malpositioning of a Matthys catheter is possible as seen in the case described later.

\section{Case Description}

A 76-year-old female patient (height: $170 \mathrm{~cm}$, weight: $60 \mathrm{~kg}$ ) was referred to us from another hospital. She had been an inpatient there for a longer period as a result of problems of wound healing following dorsal spinal fusion for a fracture of the T12 vertebra in February 2014. The metal was removed from the patient's back at the beginning of July because of problems with wound healing. After this, pleural effusion occurred on the left side. This required several punctures and at least drainage. A Matthys catheter (Matthys drainage A Set,
License terms

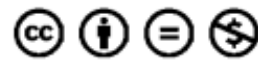

(c) 2015 Georg Thieme Verlag KG
Stuttgart · New York 10.1055/s-0035-1564691. ISSN 2194-7635.
May 20, 2015

accepted after revision

August 19, 2015

published online

October 7, 2015 


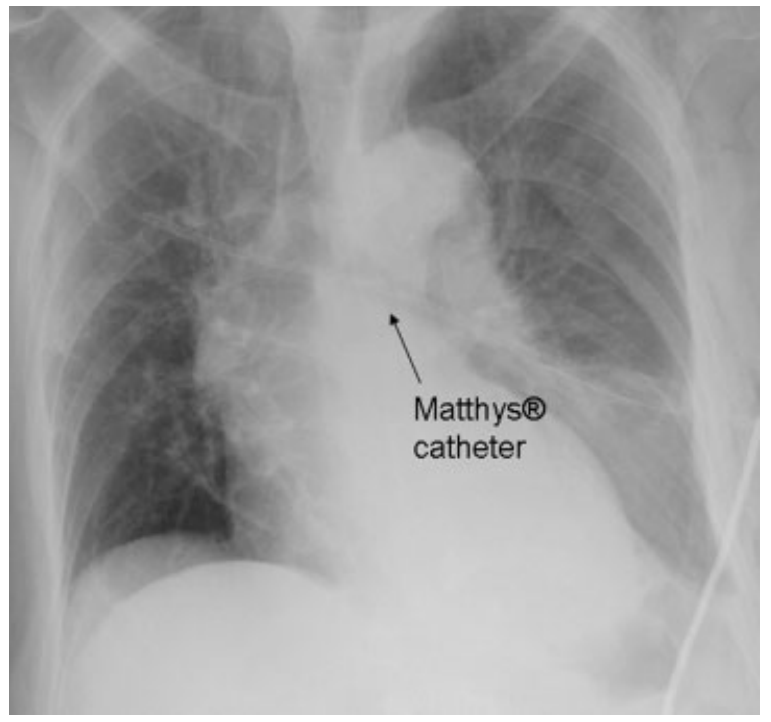

Fig. 1 X-ray of thorax directly after insertion of Matthys catheter.

Bösch Feinmechanik und Medizintechnik GmbH, Gottenheim, Germany) was inserted to drain the left-side pleural effusion.

The pleural cavity was then punctured under ultrasound control with no problem and pleural secretion was withdrawn. The pleural secretion was entirely serous, $\mathrm{pH}<7.5$. It was possible to advance the Matthys catheter with no resistance at all. Only serous pleural effusion was released into the catheter sheath. It contained no air or blood. The catheter then produced only small amounts of pleural secretion and showed no signs for an air leak. The patient did not cough once throughout the entire insertion procedure. Surprisingly, the thoracic X-ray examination revealed significant malposition of the Matthys catheter. It had crossed the pleura, entered the main bronchus via the left lung, and perforated the right upper lobe bronchus (see - Fig. 1). Pneumothorax was not apparent on either side. Computed tomography of the thorax was performed to provide further information. This revealed bleeding of the lung parenchyma on the left side along the Matthys catheter which had been inserted through the upper lobe. The catheter entered the upper lobe bronchus via a left upper lobe segment and then progressed into the left main bronchus. It crossed into the right main bronchus and then the right upper lobe bronchus and lung parenchyma of the right upper lobe (see - Fig. 2). Apart from a small apical pneumothorax on the left side, there was no soft tissue emphysema and no pneumothorax on the right side.

We then performed a bronchoscopy to confirm the catheter's suspected position as suggested by computed tomography. The endoscopic examination was performed flexibly under analgosedation. Once the endoscope reached the distal trachea, the catheter immediately became visible. It ran from the left upper lobe through the bronchial system into the right upper lobe segment (see - Fig. 3). The patient tolerated the catheter malposition without any problem. It is astonishing that there were no coughing attacks whatsoever.

Because of the small size of the Matthys catheter, we decided simply to remove it while remaining prepared to place a thoracic drain if necessary. This was achieved without problems and without any occurrence of thoracic bleeding or pneumothorax. There was no occurrence of breathlessness. We prescribed absolute bedrest for the patient. A thorax X-ray showed no pneumothorax and no soft-tissue emphysema (see - Fig. 4). The patient was transferred to the general ward 1 day later. As no lung problems occurred over the next few days, we were able to transfer the patient back to her original hospital on the 5 th day of admission.

\section{Discussion}

Matthys catheters are used very frequently, particularly in internal medicine, to release serous pleural effusions. ${ }^{3}$ The insertion process is simple. In this case, puncture and pushing of the catheter were entirely without problems. Some pleural secretion escaped when the pleural cavity was punctured, but none was released thereafter. Likewise, no bloody secretion occurred via the catheter. It was striking that, once the trap had been connected, this did not show any relation to breathing and that no air fistula occurred. After this, the
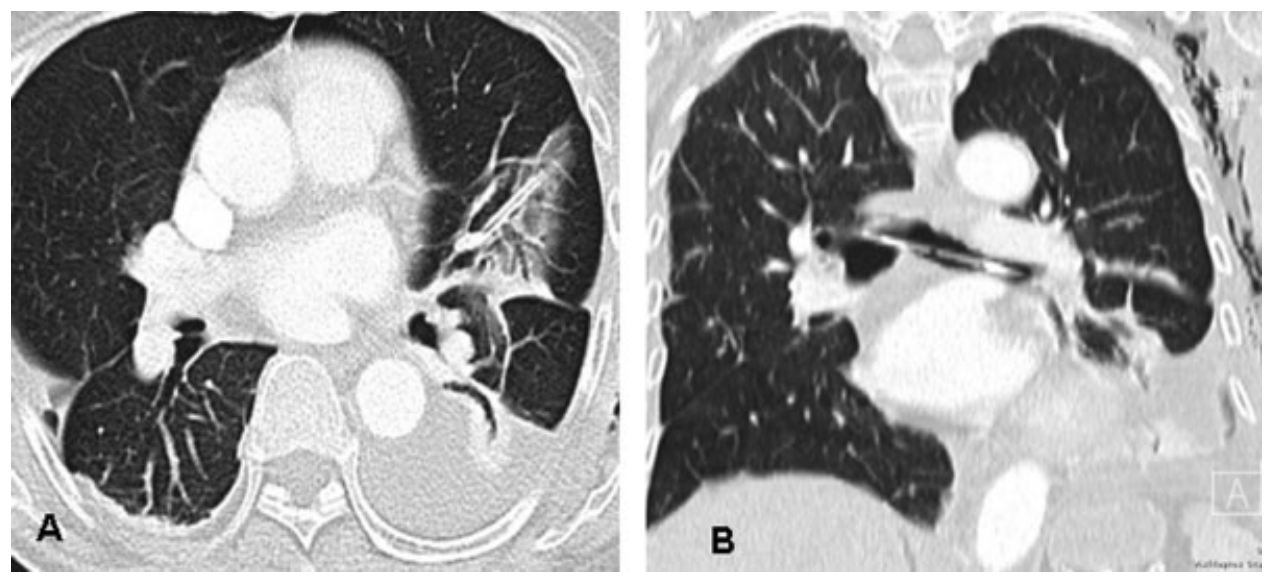

Fig. 2 Computed tomography of thorax: endobronchial malposition of the Matthys catheter: (A) bleeding of the lung parenchyma in segment 2 of the left upper lobe and persistent pleural effusion on the left side; (B) the catheter lies in the central bronchial system. 


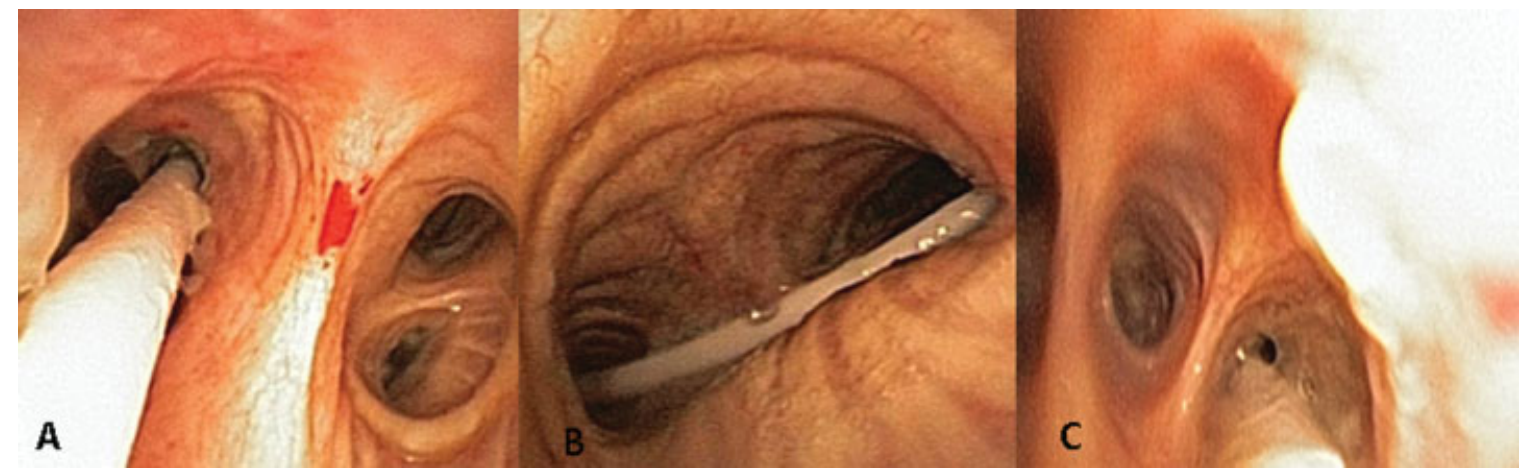

Fig. 3 Endoscopic view of the endobronchial malposition of the Matthys catheter. (A) Entrance of the Matthys catheter through the left upper lobe into the bronchial tree. (B) Bronchial bifurcation: catheter goes from the left to the right side. (C) The catheter runs into the right upper lobe.

pleural drain did not carry any pleural secretion. The thoracic $\mathrm{X}$-ray showed a malposition that has not yet been reported in the literature. During the insertion procedure, the catheter had perforated the visceral pleura and entered the bronchial system through a segmental bronchus of the left upper lobe. The bronchial system then served to guide the catheter as it was pushed ahead. It was pushed through the main bifurcation into a segmental bronchus in the right upper lobe. The diameter of the catheter was small and the entry hole in the left lung was therefore sealed over by the surrounding lung tissue. However the X-ray also showed that, in spite of ultrasound control, the catheter had been inserted much too high. The left upper lobe was probably punctured directly when the initial puncture was made. In subsequent computed tomography of the thorax, all that could be seen were a small apical pneumothorax and slight bleeding of the lung parenchyma along the drainage channel. The pleural effusion, which was actually in the left basal area, was still present because the pleural catheter had been inserted too high. In addition, the tip and perforations of the catheter had been sealed by the surrounding lung tissue. No pneumothorax occurred on the right side because the visceral pleura on that

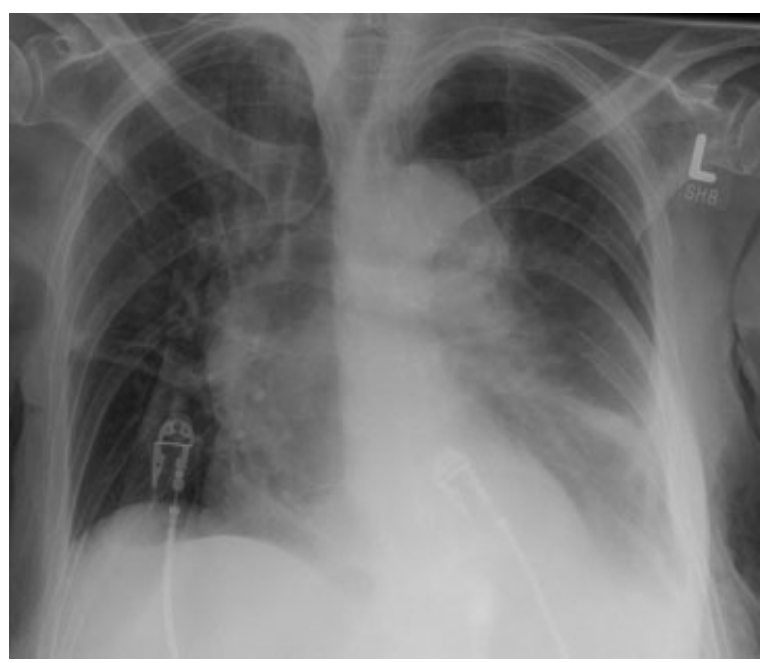

Fig. 4 Thoracic X-ray after removal of the Matthys catheter. No pneumothorax. side had not been injured. In computed tomography, the central bronchial system appeared completely intact. The patient was then transferred to our center because our colleagues feared that complications would occur when the catheter was removed.

First of all, an endoscopic evaluation of the situation inside the bronchi was indicated. Surprisingly, the patient tolerated the foreign body completely without coughing. The bronchoscopy confirmed the malposition of the catheter suggested by computed tomography. In the bronchi, no secretion or blood needed to be aspirated. It was clear that such malposition of the catheter could not be tolerated and the catheter needed to be removed immediately. In theory, bilateral pneumothorax can occur after removal. One option to consider would have been to fit the patient with prophylactic thoracic drainage on both sides to protect against bilateral tension pneumothorax. We decided against this, as the patient was awake and breathing spontaneously. We simply removed the catheter while carrying out intensive care monitoring. However, we had made preparations at the bedside for inserting thoracic drains on both sides. If respiratory insufficiency had occurred, it would have been possible to provide immediate bilateral relief even without an X-ray. If pronounced bronchopleural fistulas had been present on both sides and respiratory insufficiency had been a danger, intubation and ventilation would have been unavoidable. Ventilation with higher inspiratory pressures leads to worsening of the bronchopleural fistulas. Veno-venous extracorporeal membrane oxygenation is recommended to maintain gas exchange with implantation of cannulae via the groin.

If segmental bronchi are injured, the leaks do not seal spontaneously. Surgery is therefore the only option in this situation. As it would have been necessary to explore both lungs in a single session in this case, we would have chosen a clamshell incision for access. A lobectomy would have been unavoidable because of the diffused bleeding of the lung parenchyma along the catheter channel. On the right side, the segmental bronchus was probably injured only centrally so that the aim here would have been a segment resection.

If a massive bronchopleural fistula had occurred on the left side only, we would have performed double lumen intubation as usual, left-side thoracotomy while lying on one side, and 
then resection of the upper lobe. Luckily none of these things occurred in this case. No further thoracic problems were apparent and no secondary pneumothorax occurred. One explanation for this is the small diameter of the catheter. Even when it perforated the lung parenchyma, it created only small lesions. If thoracic drains had been inserted, such malpositioning would have had much more serious consequences. Because of its hard tip, the catheter can easily injure relatively vulnerable tissue such as lung parenchyma, especially in older patients. The position of the pleural catheter must be checked through X-ray, especially when no pleural effusion is produced. ${ }^{4}$ The Matthys catheter can also become simply kinked. This situation can be remedied very easily by withdrawing the catheter slightly.

\section{Conclusion}

Matthys catheters are often used to relieve pleural effusion or pneumothorax. In many cases, malposition can only be diagnosed by means of radiology. With endobronchial malposition, as in this case, the catheter can easily be removed without any consequences.

Conflict of Interest

The authors have nothing to declare.

\section{References}

1 Klopp M, Hoffmann H, Dienemann H. Pleural drainage [in German]. Dtsch Med Wochenschr 2009;134(11):536-539

2 Miller KS, Sahn SA. Chest tubes. Indications, technique, management and complications. Chest 1987;91(2):258-264

3 Overrath GKN, Matthys H. Anwendungsmöglichkeiten eines dünnen Saugdrain in der Pneumologie. Therapiewoche 1974; 24:1-5

4 Durai R, Hoque H, Davies TW. Managing a chest tube and drainage system. AORN J 2010;91(2):275-280, quiz 281-283 